\title{
„Meistens sind Forscher älter, meist tragen die eine Brille" - Schülervorstellungen über Wissenschaftler*innen
}

\author{
Ramona Hagenkötter $($ D $\cdot$ Valentina Nachtigall • Katrin Rolka • \\ Nikol Rummel
}

Eingegangen: 18. Dezember 2020 / Überarbeitet: 1. April 2021 / Angenommen: 28. April 2021 / Online publiziert: 10. Juni 2021

(C) Der/die Autor(en) 2021

Zusammenfassung In der vorliegenden Studie wurde mithilfe einer eigens entwickelten Fotosortierungsaufgabe die Bedeutung stereotyper Merkmale von Wissenschaftler*innen für die von Schüler*innen eingeschätzte Typikalität von Wissenschaftler*innen untersucht. Zudem wurde exploriert, wie diese Vorstellungen mit den mathematik- und naturwissenschaftsbezogenen Aktivitäten der Schüler*innen sowie ihrer Freude und ihrem Interesse an Mathematik und den Naturwissenschaften zusammenhängen. Dafür nahmen 74 Schüler*innen der Jahrgangsstufen 8 bis 10 an der Fotosortierungsaufgabe sowie an einer schriftlichen Befragung teil. Die Ergebnisse zeigen, dass aus Schülersicht vor allem folgende Merkmale typisch für Wissenschaftler*innen sind: mit Brille, älter, ohne Styling (bei Frauen) bzw. ohne Bart (bei Männern) und männlich. Ihre Auswahl begründeten die befragten Schüler*innen damit, dass Wissenschaftler*innen viel lesen müssten (Grund für Brille) und eine längere Ausbildungszeit hätten (Grund für höheres Alter). Es lassen sich zudem geschlechterspezifische Unterschiede in den Schülervorstellungen über Wissenschaftler*innen feststellen: Männliche Schüler schätzten vor allem männliche

Ramona Hagenkötter $(\bowtie) \cdot$ Katrin Rolka

Fakultät für Mathematik, Ruhr-Universität Bochum, Universitätsstr. 150, 44801 Bochum,

Deutschland

E-Mail: ramona.hagenkoetter@ruhr-uni-bochum.de

Katrin Rolka

E-Mail: katrin.rolka@ruhr-uni-bochum.de

Valentina Nachtigall · Nikol Rummel

Institut für Erziehungswissenschaft, Ruhr-Universität Bochum, Universitätsstr. 150, 44801 Bochum,

Deutschland

Valentina Nachtigall

E-Mail: valentina.nachtigall@ruhr-uni-bochum.de

Nikol Rummel

E-Mail: nikol.rummel@ruhr-uni-bochum.de 
Personen und weibliche Schülerinnen eher weibliche Personen, insbesondere Frauen mit Brille und ohne Styling, als typische Wissenschaftler*innen ein. Außerdem zeigen die Ergebnisse, dass Personen mit Bart verstärkt von Schüler*innen mit eher hohen mathematikbezogenen Aktivitäten und jüngere Personen verstärkt von Schüler*innen mit eher hoher Freude und hohem Interesse an Mathematik als typische Wissenschaftler*innen angesehen wurden. Weitere Zusammenhänge zwischen den mathematik- und naturwissenschaftsbezogenen Aktivitäten bzw. der Freude und dem Interesse der Schüler*innen an Mathematik und den Naturwissenschaften einerseits und der eingeschätzten Typikalität anderer Merkmale von Wissenschaftler*innen andererseits wurden nicht festgestellt. Die Ergebnisse werden mit bisherigen Forschungsbefunden verglichen und Möglichkeiten zur Verringerung solch stereotyper Wahrnehmungen diskutiert.

Schlüsselwörter Schülervorstellungen über Wissenschaftler*innen · Stereotype · Wissenschaftsbezogene Aktivitäten · Freude und Interesse an Wissenschaft

\title{
"Scientists are usually older and wear glasses"-Students' conceptions about scientists
}

\begin{abstract}
In this study, we used a self-developed photo-sorting task in order to investigate the role of stereotypical characteristics of scientists for students' perceived typicality of scientists. In addition, we explored the relation between these beliefs and students' mathematics and science-related activities as well as their enjoyment and interest in mathematics and the natural sciences. For this purpose, 74 th to 10 th graders worked on the photo-sorting task and filled in a questionnaire. The results show that the majority of the students associated the following characteristics with scientists: with glasses, older, without styling (for women) or without beard (for men), and male. Students often justified these choices by pointing out that scientists have to read a lot (which is why they wear glasses) and that they have to go through a long education (which is the reason for their older age). Our results also demonstrate gender differences in students' conceptions of scientists: While male students tend to associate men with typical scientists, female students more likely associate women, especially women with glasses and without styling, with typical scientists. Furthermore, our analyses reveal that students who reported a rather high level of mathematics-related activities more likely tend to relate people with beard. Students who reported a rather high level of enjoyment and interest in mathematics more likely tend to relate younger people with typical scientists. We found no further relations between students' mathematics and science-related activities or their enjoyment and interest in mathematics and the natural sciences on the one hand and their perceived typicality of other characteristics of scientists on the other hand. We discuss the results in light of previous research and derive ideas for reducing such stereotypical perceptions in students.
\end{abstract}

Keywords Students' conceptions about scientists · Stereotypes · Science-related activities - Enjoyment and interest in science 


\section{Einleitung}

Bei Schüler*innen unterschiedlichen Alters und kulturellen Hintergrunds lassen sich stereotype Vorstellungen über Wissenschaft und Wissenschaftler*innen feststellen (z.B. Chambers 1983; Höttecke 2001; Christidou et al. 2012). Die Begriffe „Wissenschaft" und „Wissenschaftler*innen“ werden dabei meist mit MINT-Disziplinen und naturwissenschaftlichen Arbeitsweisen assoziiert (vgl. Nachtigall et al. 2018; Ziegler et al. 2018; Stamer et al. 2019). Beispielsweise zeigen die Ergebnisse des Wissenschaftsbarometers 2017 (Ziegler et al. 2018), dass die Befragten vor allem auch Themen aus den Naturwissenschaften mit „Wissenschaft“ oder „Forschung“ verbanden, wohingegen Themen aus sozial- und geisteswissenschaftlichen Disziplinen nur sehr selten mit „Wissenschaft“ assoziiert wurden. Ein ähnliches Ergebnis offenbarte sich auch in der Studie von Nachtigall et al. (2018), in der sich die Teilnehmer*innen eines geisteswissenschaftlichen Schülerlaborprojekts vorwiegend Personen aus der Wissenschaft als Naturwissenschaftler*innen vorstellten.

Die Vorstellungen von Schüler*innen über Wissenschaft und Wissenschaftler*innen beeinflussen ihre Einstellungen gegenüber Wissenschaft (d.h. ihre Gefühle, Überzeugungen und Werte in Bezug auf Wissenschaft; vgl. Osborne et al. 2003) stark (z.B. Boylan et al. 1992; Finson 2002; Buldu 2006). Schülervorstellungen über Naturwissenschaftler*innen machen einen Teil ihres Verständnisses über die Natur der Naturwissenschaften aus (vgl. Höttecke 2001). Bisherige Studien über Vorstellungen von Schüler*innen über die Natur der Naturwissenschaften adressierten überwiegend die epistemologischen Überzeugungen von Schüler*innen sowie ihre Vorstellungen über konkrete Tätigkeits- oder typische Arbeitsfelder von Naturwissenschaftler*innen (z. B. Wentorf et al. 2015; Stamer et al. 2019). Dabei stellen jedoch nicht nur die Vorstellungen über typische Tätigkeits- oder Arbeitsfelder von Wissenschaftler*innen eine Basis für die Berufsorientierung von Schüler*innen dar (vgl. Wentorf et al. 2015). Hannover und Kessels (2002) konnten beispielsweise - in Anlehnung an den „Selbst-Prototypen-Abgleich“ (Setterlund und Niedenthal 1993) - zeigen, dass Schüler*innen ihre schulischen Präferenzen und Wahlen danach ausrichteten, wie ähnlich oder unähnlich sie ihr Selbst zu prototypischen Personen sahen, die Physik präferierten bzw. den Beruf des Ingenieurs ergriffen. Auch Buldu (2006) konnte in einer Interview-Studie eine Verbindung zwischen den Vorstellungen von Schüler*innen über Wissenschaftler*innen und ihrem Interesse an wissenschaftlichen Berufen feststellen: Teilweise wiesen die Schüler*innen, als sie ihr Bild von einem*einer Wissenschaftler*in zeichneten, darauf hin, dass sie in der Zukunft der*die gezeichnete Wissenschaftler*in sein wollten. Folglich kann davon ausgegangen werden, dass vor allem auch die Vorstellungen von Schüler*innen über die konkrete Person eines*einer Wissenschaftler*in und deren Aussehen eine wichtige Rolle für zukünftige Bildungs- und Berufsentscheidungen spielen: Sehen sich die Schüler*innen selbst nicht als potenzielle Wissenschaftler*innen, könnte das nicht nur ihr Interesse an Wissenschaft verringern, sondern vor allem auch ihre zukünftigen Bildungs- und Berufswege beeinflussen (vgl. Laubach et al. 2012; Stamer et al. 2019). Vor allem im Hinblick auf die Generierung wissenschaftlichen Nachwuchses scheint es daher notwendig, die Vorstellungen von Schüler*innen über Wissenschaftler*innen näher zu untersuchen, um anschließend Maßnahmen zur ge- 
zielten Förderung authentischer Schülervorstellungen über Wissenschaftler*innen ableiten zu können.

Die bisher angewendeten Methoden zur Erfassung von Schülervorstellungen über Wissenschaftler*innen sind jedoch begrenzt und starker Kritik unterworfen (vgl. Finson 2002). Vor diesem Hintergrund zielt die vorliegende Studie darauf ab, die Vorstellungen von Schüler*innen über Wissenschaftler*innen zu untersuchen, indem aus Schülersicht besonders typische äußerliche Merkmale von Wissenschaftler*innen identifiziert werden. Dazu wurde mithilfe einer eigens entwickelten Fotosortierungsaufgabe die Bedeutung einzelner und auf Grundlage bisheriger Befunde ausgewählter stereotyper Merkmale von Wissenschaftler*innen für die von Schüler*innen eingeschätzte Typikalität von Wissenschaftler*innen untersucht. Die vorliegende Studie exploriert außerdem, wie die Schülervorstellungen über Wissenschaftler*innen mit den mathematik- und naturwissenschaftsbezogenen Aktivitäten der Schüler*innen in oder außerhalb der Schule, wie beispielsweise Fernsehsendungen über mathematische/naturwissenschaftliche Themen schauen, sowie ihrer Freude und ihrem Interesse an Mathematik und den Naturwissenschaften zusammenhängen.

\section{Theoretischer Hintergrund}

\subsection{Schülervorstellungen über Wissenschaftler*innen}

Eine erste großangelegte Untersuchung zu Vorstellungen von Schüler*innen über Wissenschaftler*innen wurde von Mead und Métraux (1957) in den USA durchgeführt. Die Ergebnisse ihrer Aufsatzstudie zeigten, dass die meisten Schüler*innen über stereotype Vorstellungen über Wissenschaftler*innen verfügten und diese als männliche Personen älteren oder mittleren Alters beschrieben, die einen Laborkittel sowie eine Brille tragen, und alleine in einem Laboratorium Experimente durchführen. Ähnliche stereotype Vorstellungen über Wissenschaftler*innen offenbarten sich auch in der Studie von Chambers (1983), an der Kinder aus Kanada, den USA und Australien vom Kindergartenalter bis zur 5. Klasse teilnahmen. Die Studie wurde mit dem Draw-A-Scientist Test (DAST) durchgeführt. Die Ergebnisse zeigten, dass insbesondere Laborkittel, Brille und Gesichtsbehaarung in den Zeichnungen vorkamen. Stereotype, die in verschiedenen DAST-Studien identifiziert wurden, sind bei Hassard (1990) zusammengefasst: Bei den gezeichneten Personen handelt es sich in der Regel um einen männlichen Kaukasier, der entweder kahlköpfig oder mit krausem, wildem Haar dargestellt ist. Außerdem trägt der Wissenschaftler eine Brille und ist mit einem weißen Laborkittel bekleidet. Er arbeitet alleine in einem Labor. Der Wissenschaftler mischt Chemikalien, führt eine Art chemie- oder physikbezogenes Experiment durch, oder experimentiert mit gefährlichen Dingen und manchmal an Menschen. Auch die Ergebnisse der Studie von Boylan et al. (1992), in der Schüler*innen aus Malaysia Illustrationen von Wissenschaftler*innen auswählen und ihre Auswahl begründen sollten, zeigten, dass sich die meisten Schüler*innen Wissenschaftler*innen als bärtige Männer vorstellen, die eine Brille und einen Laborkittel tragen. Wissenschaftler*innen wurden dabei von den Proband*innen ebenfalls als äl- 
ter angesehen, während jüngere Personen oftmals von den befragten Schüler*innen als Lernende oder Wissenschaftler*innen in Ausbildung beschrieben wurden. Bei den Begründungen ihrer Auswahl stellten die Proband*innen außerdem oft direkte Verbindungen zu ihren eigenen Naturwissenschaftslehrer*innen her, die von ihnen als typische Wissenschaftler*innen angesehen wurden.

In allen bereits erwähnten Studien war das Geschlecht „männlich“ ein Attribut der stereotypen Vorstellungen der Kinder. Ähnliche Ergebnisse wurden auch in weiteren Studien gefunden, beispielsweise von Fort und Varney (1989) und von Christidou et al. (2012). In der Studie von Chambers (1983) zeichneten tatsächlich nur 28 von 4807 Kindern $(0,6 \%)$ eine weibliche Wissenschaftlerin, wobei weibliche Wissenschaftlerinnen ausschließlich von weiblichen Probandinnen gezeichnet wurden. Die Ergebnisse von Christidou et al. (2012) zeigten ebenfalls, dass männliche Schüler eher männliche Wissenschaftler zeichneten, wohingegen weibliche Schülerinnen häufiger weibliche Wissenschaftlerinnen zeichneten. Miller et al. (2018) kamen in ihrer Meta-Analyse, die DAST-Studien zu stereotypen Vorstellungen von Kindern über Wissenschaftler*innen in den USA über fünf Jahrzehnte berücksichtigt, ebenfalls zu einem ähnlichen geschlechterspezifischen Ergebnis: So zeichneten in mehr als der Hälfte der untersuchten Studien männliche Probanden ausschließlich männliche Wissenschaftler.

Wie die Ergebnisse bisheriger Studien verdeutlichen, sind die Vorstellungen von Schüler*innen über Wissenschaftler*innen von Stereotypen geprägt (u. a. Mead und Métraux 1957; Chambers 1983; Hassard 1990; Boylan et al. 1992). Oftmals wird an vielen der exemplarisch beschriebenen Studien zu Schülervorstellungen über Wissenschaftler*innen jedoch die Methodik kritisiert.

\subsection{Erfassung von Schülervorstellungen über Wissenschaftler*innen}

In der Studie von Mead und Métraux (1957) wurden die Schülervorstellungen über Wissenschaftler*innen in schriftlicher Form eines Essays erfasst. Die Erfassung der Schülervorstellungen in schriftlicher Form stellt jedoch hohe sprachliche Anforderungen an die Proband*innen und die Auswertung ist oftmals zeitintensiv. Im Gegensatz dazu sind bei dem von Chambers (1983) entwickelten DAST keine verbalen Fähigkeiten erforderlich und die Beurteilung ist wenig zeitintensiv (Finson et al. 1995). In diesem später vielfach verwendeten Format stellen die Proband*innen ihre Vorstellungen zeichnerisch dar, womit die Ergebnisse zwar nicht von den sprachlichen aber den zeichnerischen Fähigkeiten der Proband*innen abhängen (Losh et al. 2008). Gleichwohl existieren jedoch auch Studien, in denen dieser Kritik entgegengewirkt wird und mögliche zeichnerische Hemmschwellen der Proband*innen abgeschwächt werden, indem sie zunächst vertraute Figuren, beispielsweise eine Lehrkraft, zeichnen (z.B. Losh et al. 2008). Darüber hinaus wird auch die Art und Weise, wie die Anweisungen für den DAST gegeben werden, kritisiert. So könnte die Art der Anweisungen bereits vorherrschende Stigmatisierungen und Vorurteile provozieren (Wentorf et al. 2015) und suggerieren, dass es so etwas wie eine*n typische*n Wissenschaftler*in gibt und dass die Proband*innen ein solches Einzelbild darstellen sollen (McNay 1988). Diese Kritik wurde in verschiedenen modifizierten Varianten aufgegriffen. In einigen anderen DAST-Studien mussten die 
Teilnehmer*innen beispielsweise mehrere Bilder von Wissenschaftler*innen zeichnen, was nach der ersten Zeichnung häufiger zu Wissenschaftlerinnen führte (Losh et al. 2008). Auch Farland-Smith (2012) modifizierte den DAST und forderte die Proband*innen dazu auf, das Aussehen, den Ort sowie die Tätigkeiten von Wissenschaftler*innen zu zeichnen (mDAST). Außerdem sollten schriftliche Angaben, beispielsweise zum Geschlecht der gezeichneten Person, gemacht werden.

Allerdings offenbaren der DAST und auch die modifizierten DAST-Varianten möglicherweise nicht die gesamte Bandbreite der Schülervorstellungen über Wissenschaftler*innen (Finson et al. 1995; Finson und Pederson 2011). Um ein tieferes Verständnis über solche Vorstellungen entwickeln zu können, verwendeten beispielsweise Boylan et al. (1992) in ihrer Studie das Interview-about-instances-Verfahren. Dafür nutzte die Studie 30 Illustrationspaare (z. B. männlicher Kaukasier mit Laborkittel und weibliche Kaukasierin mit Laborkittel) sowie offene Fragen (z. B. „Is one of the persons more likely to be a scientist than the other or could they both be scientists or could neither be a scientist-why?"), welche die Bereiche Aussehen, Arbeitsplatz, Arbeitsaufgabe und Beschäftigung von Wissenschaftler*innen adressierten. Damit sind die Ergebnisse nicht mehr von den zeichnerischen Fähigkeiten der Proband*innen abhängig und den Schüler*innen wird die Möglichkeit gegeben, ihre Auswahl zu begründen. Mithilfe der Illustrationspaare variierten Boylan et al. (1992) bestimmte Merkmale von Wissenschaftler*innen und konnten damit untersuchen, welche Merkmale die Proband*innen mit Wissenschaftler*innen assoziierten. Allerdings fand diese Variation der Merkmale eher unsystematisch statt. So nutzen sie beispielsweise das Illustrationspaar Mann mit Brille versus junger Mann ohne Brille. Die Auswahl einer der beiden Illustrationen durch die Proband*innen erlaubt jedoch keine Rückschlüsse darauf, welche Rolle die beiden gleichzeitig variierten Merkmale Alter und Brille für die Auswahl des Bildes und damit für die Einschätzung der Typikalität eines*einer Wissenschaftler*in spielen. Basierend auf der Auswahl kann nicht festgestellt werden, welcher der beiden variierten Faktoren ausschlaggebender für die Schüler*innen war, um die dargestellte Person eher als typische*n Wissenschaftler*in anzusehen. Um untersuchen zu können, welche spezifischen Merkmale aus Schülersicht typisch für Wissenschaftler*innen sind, ist daher eine systematische Variation dieser Merkmale notwendig.

Zusammenfassend lässt sich festhalten, dass Schülervorstellungen über Wissenschaftler*innen bisher häufig mit dem DAST oder modifizierten DAST-Varianten erfasst wurden. Diese und ähnliche Methoden sind jedoch aus den zuvor genannten Gründen kritisch zu bewerten. Die von Boylan et al. (1992) verwendete Interviewabout-instances-Methodik stellt einen ersten Schritt in Richtung einer zuverlässigeren Erfassung von Schülervorstellungen über Wissenschaftler*innen dar. Bislang mangelt es aber an Messverfahren, die Aufschluss über die Bedeutung einzelner Merkmale für die von Schüler*innen eingeschätzte Typikalität von Wissenschaftler*innen geben können.

\subsection{Weitere interessierende Variablen}

Wie bereits erwähnt, wurden in bisherigen Studien geschlechterspezifische Unterschiede in den Schülervorstellungen über Wissenschaftler*innen festgestellt (z.B. 
Chambers 1983; Fort und Varney 1989; Christidou et al. 2012; Miller et al. 2018). Abgesehen vom Geschlecht der Schüler*innen gibt es in der Literatur zahlreiche Annahmen, aber bislang nur vereinzelte empirische Studien, von welchen weiteren Variablen die Vorstellungen über Wissenschaftler*innen beeinflusst werden können. So wird angenommen, dass beispielsweise Alltagserfahrungen der Schüler*innen mit Wissenschaft, aber auch Medien wie Film, Fernsehen und Comics ihre Vorstellungen über Wissenschaftler*innen beeinflussen (vgl. Driver et al. 1996; Thomson et al. 2019). Thomson et al. (2019) untersuchten beispielsweise in ihrer DASTStudie, welchen Einfluss frühere Besuche von Wissenschaftsmuseen auf die Vorstellungen der Schüler*innen über Wissenschaftler*innen haben. Die Ergebnisse zeigten, dass frühere Museumsbesuche stereotype Vorstellungen begünstigen: Schüler*innen, die in der Umfrage angaben, Wissenschaftsmuseen besucht zu haben, erzielten signifikant höhere Stereotypisierungsindikatoren als Schüler*innen, die keine Wissenschaftsmuseen besucht hatten. Thomson et al. (2019) vermuten, dass sich die besuchten Museen auf die Präsentation von Wissenschaftler*innen aus vergangenen Jahrhunderten konzentriert haben könnten, sodass vorwiegend weiße Männer als Wissenschaftler dargestellt wurden, was dazu beigetragen haben könnte, die stereotypen Vorstellungen der Schüler*innen über Wissenschaftler*innen zu unterstützen. Die Studie von Thomson et al. (2019) liefert damit erste Hinweise darauf, dass Schülervorstellungen über Wissenschaftler*innen durch frühere wissenschaftsbezogene Aktivitäten der Schüler*innen (hier: Besuche von Wissenschaftsmuseen) beeinflusst werden können. Das Bild von Wissenschaft und Wissenschaftler*innen wird jedoch nicht nur durch Museen geprägt. Auch Medien und die mediale Berichterstattung können einen Einfluss auf die Vorstellungen haben (vgl. Driver et al. 1996; Buldu 2006; Ziegler et al. 2018; Thomson et al. 2019). So zeigte sich beispielsweise in der Studie von Buldu (2006), dass Schülervorstellungen über Wissenschaft oftmals durch Fernsehdarstellungen beeinflusst wurden und dass viele Schüler*innen das im Fernsehen Gesehene in ihren Zeichnungen von Wissenschaftler*innen verwendeten.

In der Literatur wird außerdem oftmals angenommen, dass die Vorstellungen über Wissenschaftler*innen die naturwissenschaftlichen Interessen der Schüler*innen beeinflussen (z. B. Boylan et al. 1992; Christidou 2011) und daher eine wichtige Rolle bei der Entwicklung ihres naturwissenschaftlichen Interesses spielen (z. B. Mead und Métraux 1957; Wentorf et al. 2015). Die Förderung motivationaler Orientierungen ist neben dem Wissens- und Kompetenzzuwachs ein wichtiges Ziel naturwissenschaftlichen Unterrichts, denn: „Wissen allein hilft im Alltag wenig, wenn Schülerinnen und Schüler sich nicht für Naturwissenschaften interessieren oder ihre Bedeutung für den Alltag oder das Weltgeschehen nicht nachvollziehen können“" (Schiepe-Tiska et al. 2016b, S. 55). Obwohl dieser Zusammenhang zwischen Vorstellungen der Schüler*innen über Wissenschaftler*innen und ihrem Interesse an Wissenschaft bislang empirisch noch nicht untersucht wurde, wurden Interventionen zur Förderung angemessener Vorstellungen über Wissenschaftler*innen entwickelt, um die Freude und das Interesse von Schüler*innen an Naturwissenschaften zu wecken. Beispielsweise wurde auf einen Abbau von Stereotypisierungen von Schulfächern fokussiert, um insbesondere einer eher männlich konnotierten Wahrnehmung von Physik und Technik zu begegnen (vgl. Labudde und Möller 2012; Schiepe-Tiska et al. 2016c). 
Zusammenfassend gibt es in der Literatur zwar zahlreiche Annahmen, mit welchen weiteren Variablen - neben dem Geschlecht der Schüler*innen - die Vorstellungen über Wissenschaftler*innen zusammenhängen, allerdings wurden diese angenommenen Zusammenhänge bislang noch nicht (ausreichend) empirisch untersucht. Thomson et al. (2019) konnten in ihrer Studie zwar erste Hinweise liefern, dass frühere wissenschaftsbezogene Aktivitäten die Vorstellungen über Wissenschaftler*innen beeinflussen, allerdings sind weitere empirische Untersuchungen der vielfach angenommenen Zusammenhänge notwendig, um konkrete Ansatzpunkte und begründete Interventionen zur Überwindung stereotyper Sichtweisen identifizieren zu können.

\subsection{Forschungsfragen}

Vor dem Hintergrund des Einflusses stereotyper Schülervorstellungen über Wissenschaftler*innen auf zukünftige Bildungs- und Berufswahlen der Schüler*innen (z. B. Boylan et al. 1992; Finson 2002; Buldu 2006; Laubach et al. 2012; Stamer et al. 2019) sowie der methodischen Kritik an bisherigen Studien zu Schülervorstellungen über Wissenschaftler*innen soll in der vorliegenden Studie systematisch die Bedeutung einzelner, auf Grundlage bisheriger Befunde ausgewählter Merkmale für die von Schüler*innen eingeschätzte Typikalität von Wissenschaftler*innen untersucht werden (Forschungsfrage 1). Ähnlich wie in der Studie von Boylan et al. (1992) soll nicht nur untersucht werden, welche Merkmale typisch für Wissenschaftler*innen sind, sondern auch wie Schüler*innen die Auswahl bestimmter, aus ihrer Sicht für Wissenschaftler*innen typischer Merkmale begründen (Forschungsfrage 2). Ausgehend von den Befunden zum Einfluss des Geschlechts von Schüler*innen auf ihre Vorstellungen über Wissenschaftler*innen (z. B. Chambers 1983; Fort und Varney 1989; Christidou et al. 2012; Miller et al. 2018) soll außerdem überprüft werden, ob sich geschlechterspezifische Unterschiede in den Schülervorstellungen über Wissenschaftler*innen feststellen lassen (Forschungsfrage 3). Da „Wissenschaft“ und „Wissenschaftler*innen" meist mit MINT und naturwissenschaftlichen Arbeitsweisen assoziiert werden (vgl. Nachtigall et al. 2018; Ziegler et al. 2018; Stamer et al. 2019), soll in der vorliegenden Studie - basierend auf den Ergebnissen von Thomson et al. (2019) und Buldu (2006), die darauf hindeuten, dass frühere wissenschaftsbezogene Aktivitäten von Schüler*innen ihre Vorstellungen über Wissenschaftler*innen beeinflussen - zudem ein möglicher Zusammenhang zwischen den Vorstellungen von Schüler*innen über Wissenschaftler*innen und ihren mathematik- und naturwissenschaftsbezogenen Aktivitäten exploriert werden (Forschungsfrage 4). Vor dem Hintergrund der vielfach in der Literatur formulierten (z. B. Boylan et al. 1992; Christidou 2011), aber bisher kaum untersuchten Annahme, dass Schülervorstellungen über Wissenschaftler*innen unter anderem mit ihren naturwissenschaftsbezogenen Interessen zusammenhängen, soll auch untersucht werden, ob und inwiefern die Vorstellungen von Schüler*innen über Wissenschaftler*innen mit ihrer Freude und ihrem Interesse an Mathematik und den Naturwissenschaften zusammenhängen (Forschungsfrage 5). Die Bezeichnung „Freude und Interesse an“ wurde dabei aus bisherigen PISA-Studien - unter anderem PISA 2015 (vgl. Mang et al. 2019, S. 90) - übernommen. 


\section{Methode}

\subsection{Stichprobe}

Zur Untersuchung der beschriebenen Forschungsfragen wurde eine empirische Untersuchung mit 74 Schüler*innen der Jahrgangsstufen 8 bis 10 (62,2\% männlich, $35,1 \%$ weiblich, $1,4 \%$ divers, $M=14,97$ Jahre, $S D=1,33)$ durchgeführt. Die Studie fand im Februar und März 2020 im Rahmen von fünf verschiedenen natur- und geisteswissenschaftlichen Projekttagen im Alfried Krupp-Schülerlabor der RuhrUniversität Bochum statt.

\subsection{Design}

Mithilfe einer Fotosortierungsaufgabe wurden in einem Within-Subject Design äußerliche Eigenschaften von Wissenschaftler*innen systematisch variiert und der Einfluss dieser Merkmale auf die von den Schüler*innen getroffene Einschätzung der Typikalität von Wissenschaftler*innen untersucht (siehe Forschungsfrage 1). Basierend auf bisherigen Forschungsbefunden (u.a. Mead und Métraux 1957; Chambers 1983; Hassard 1990; Boylan et al. 1992) fand eine Variation folgender Faktoren statt: Geschlecht (männlich/weiblich), Alter (älter/jünger), Brille (ohne/mit Brille) sowie bei den Männern Bart (ohne/mit Bart) und bei den Frauen Styling (ohne/mit Schminke und Kette) (siehe Tab. 1).

Zudem fanden eine mündliche und eine schriftliche Befragung der Schüler*innen statt, um die Forschungsfragen 2-5 zu untersuchen.

\subsection{Material}

Die Variation der ausgewählten Faktoren wurde mithilfe von Portraitfotos umgesetzt. Die Faktoren Geschlecht und Alter wurden dabei durch Portraitfotos von vier unterschiedlichen Personen (älterer Mann, jüngerer Mann, ältere Frau und jüngere Frau) variiert. Die Faktoren Brille und bei Männern Bart bzw. bei Frauen Styling wurden hingegen innerhalb der jeweiligen Portraitfotos der vier Personen variiert. Demnach wurden von jeder der vier Personen insgesamt vier verschiedene Portraitfotos (d.h. mit Brille und mit Bart bzw. Styling, ohne Brille und mit Bart bzw. Styling, mit Brille und ohne Bart bzw. Styling, ohne Brille und ohne Bart bzw. Styling) erstellt. Um die Variation der Faktoren Brille und Bart bzw. Styling über die vier verschiedenen Personen gleich zu halten, bekamen alle Personen dieselbe Brille aufgesetzt und der gleiche Bart bzw. die gleiche Kette wurden nachträglich mithilfe eines Bildbearbeitungsprogrammes angebracht. Abb. 1 zeigt beispielhaft die vier Portraitfotos einer jüngeren Frau, wie sie in ähnlicher Form in der Studie verwendet

Tab. 1 Variierte Faktoren und deren Faktorstufen

\begin{tabular}{llllll}
\hline Faktor & Geschlecht & Alter & Brille & Bart & Styling \\
\hline Merkmale & männlich & älter & ohne Brille & ohne Bart & ohne Kette und Schminke \\
& weiblich & jünger & mit Brille & mit Bart & mit Kette und Schminke \\
\hline
\end{tabular}



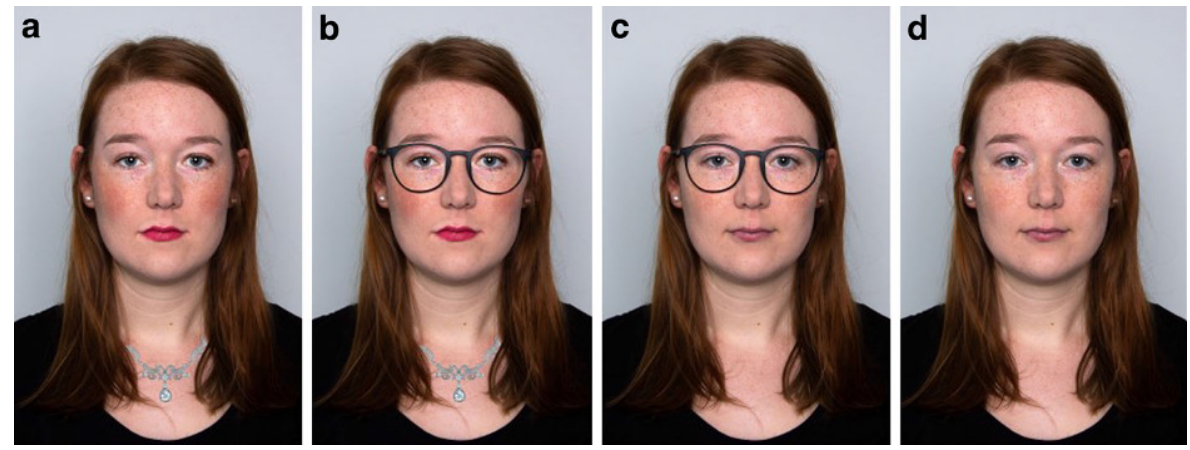

Abb. 1 Beispielhafte Portraitfotos einer jüngeren Frau (Faktoren Geschlecht („weiblich“) und Alter (,jünger") konstant, Faktoren Brille und Styling variiert). a jüngere Frau ohne Brille und mit Styling, b mit Brille und mit Styling, $\mathbf{c}$ mit Brille und ohne Styling, $\mathbf{d}$ ohne Brille und ohne Styling

wurden. Insgesamt wurden 16 Fotos (jeweils vier Fotos eines älteren Mannes, eines jüngeren Mannes, einer älteren Frau sowie einer jüngeren Frau) eingesetzt.

\subsection{Instrumente}

Um die aus Schülersicht typischen Merkmale von Wissenschaftler*innen zu untersuchen (Forschungsfrage 1), wurde eine Fotosortierungsaufgabe entwickelt. Zu Beginn der Fotosortierungsaufgabe bekamen die Proband*innen nacheinander jeweils die vier Portraitfotos des älteren Mannes, des jüngeren Mannes, der älteren Frau sowie der jüngeren Frau gezeigt (d.h. insgesamt 16 Fotos). Die Aufgabe der Proband*innen war es in einem ersten Schritt, die vier Fotos derselben Person hinsichtlich der Frage „Auf welchem Foto sieht die Person für dich am ehesten wie ein/e Wissenschaftler/in aus?" (Position 1: „am ehesten“; Position 4: „am wenigsten“) zu sortieren. In einem zweiten Schritt sahen die Proband*innen noch einmal diejenigen Fotos, die sie im ersten Schritt jeweils auf die ersten beiden Positionen einer Person sortiert hatten (d.h. die jeweils auf den ersten beiden Positionen sortierten Fotos des älteren Mannes, des jüngeren Mannes, der älteren Frau sowie der jüngeren Frau). Aus diesen insgesamt acht Fotos sollten die Schüler*innen ihre Top 4 Fotos (beginnend mit ihrem Platz 1) auswählen, auf denen die abgebildeten Personen für sie am ehesten wie Wissenschaftler*innen aussahen (Platz 1 $\hat{=} 4$ Punkte, Platz $2 \hat{=} 3$ Punkte, Platz $3 \hat{=} 2$ Punkte, Platz $4 \hat{=} 1$ Punkt und nicht in die Top 4 gewählte Fotos $\hat{=} 0$ Punkte). Zur Identifizierung der typischen Merkmale von Wissenschaftler*innen aus Schülersicht wurden anschließend die Typikalitätswerte der einzelnen Merkmale der Top 4 Fotos summiert $(0 \hat{=}$,Das entsprechende Merkmal ist auf keinem der in die Top 4 gewählten Fotos vertreten“ bis $10 \hat{=}$,Das entsprechende Merkmal ist auf allen in die Top 4 gewählten Fotos vertreten“). Sortiert beispielsweise ein*e Schüler*in auf alle Top 4 Plätze Fotos von Personen mit Brille, so erzielt das Merkmal „,mit Brille“ insgesamt einen Typikalitätswert von 10 und wird somit sehr typisch für Wissenschaftler*innen eingeschätzt.

Um zu untersuchen, wie Schüler*innen die Auswahl bestimmter, aus ihrer Sicht für Wissenschaftler*innen typischer Merkmale begründen (Forschungsfrage 2), wur- 
den einzelne Schüler*innen ausgewählt und in Form eines leitfadengestützten Interviews mündlich zu den Gründen ihrer Top 4 Fotoauswahl befragt. Den Schüler*innen wurden die folgenden zwei Fragen gestellt: „Auf welche Merkmale hast du bei deiner Zuordnung bzw. Sortierung geachtet? “ und „, Warum hast du auf diese Merkmale geachtet?". Die Antworten der Schüler*innen wurden von der Interviewerin im Wortlaut schriftlich festgehalten.

Zur Erfassung des Geschlechts der Schüler*innen (siehe Forschungsfrage 3), der mathematik- und naturwissenschaftsbezogenen Aktivitäten (siehe Forschungsfrage 4) sowie der Freude und des Interesses an Mathematik und den Naturwissenschaften (siehe Forschungsfrage 5) wurde ein Fragebogen eingesetzt. Zur Erfassung der mathematik- und naturwissenschaftsbezogenen Aktivitäten wurden die Schüler*innen gebeten anzugeben, wie oft sie an sechs ausgewählten Aktivitäten in oder außerhalb der Schule teilnehmen (Mathematik: Cronbach's $\alpha=0,81$; Naturwissenschaften: Cronbach's $\alpha=0,86$ ), beispielsweise „Fernsehsendungen über mathematische/naturwissenschaftliche Themen anschauen“. Die Einschätzungen erfolgten auf einer fünfstufigen Antwortskala: 1=,Fast nie“ bis 5=,Sehr oft“. Die Freude und das Interesse an Mathematik sowie den Naturwissenschaften wurden jeweils mittels fünf Aussagen erhoben (Mathematik: Cronbach's $\alpha=0,89$; Naturwissenschaften: Cronbach's $\alpha=0,92$ ), beispielsweise ,Im Allgemeinen macht es mir Spaß, mich mit mathematischen/naturwissenschaftlichen Themen zu befassen." Die Einschätzungen erfolgten ebenfalls auf einer fünfstufigen Antwortskala: 1 =,Stimme überhaupt nicht zu“ bis 5= „Stimme völlig zu“. Die vier verwendeten Fragebogenskalen wurden aus PISA 2015 (vgl. Mang et al. 2019) adaptiert. Die internen Konsistenzen sind laut Cicchetti und Sparrow (1990) zufriedenstellend.

\subsection{Durchführung}

Für die Teilnahme an der Studie wurden die Schüler*innen in Kleingruppen (maximal acht Schüler*innen) aus den laufenden Projekten in einen separaten Raum im Schülerlabor gebeten. Die Zeitpunkte variierten dabei je nach Projekt, da die Erhebungen den Projektablauf möglichst wenig stören sollten. Nach einer kurzen Instruktion bearbeiteten die Schüler*innen in einem ersten Schritt an Laptops und Tablets des Schülerlabors die Fotosortierungsaufgabe. Um Reihenfolgeeffekte zu vermeiden, variierte zwischen den Proband*innen sowohl die Reihenfolge der Fotos der vier Personen (d.h. älterer Mann, jüngerer Mann, ältere Frau und jüngere Frau) als auch die Reihenfolge der vier Fotos pro Person nach dem Zufallsprinzip. Im zweiten Schritt beantworteten die Schüler*innen den Fragebogen. Die Bearbeitungszeit betrug insgesamt ungefähr 15 min. Abschließend wurden einzelne Schüler*innen ausgewählt und mündlich zu den Gründen ihrer Top 4 Fotoauswahl befragt. Um ein möglichst differenziertes Bild der Gründe für die Auswahl bestimmter Merkmale von Wissenschaftler*innen aus Schülersicht zu gewinnen, wurden dabei sowohl Schüler*innen ausgewählt, deren Fotoauswahl die Ergebnisse aus der Literatur widerspiegeln (d.h. stereotype Vorstellungen über Wissenschaftler*innen), als auch Schüler*innen, die über weniger stereotype Vorstellungen über Wissenschaftler*innen verfügten bzw. eine besondere Auswahl trafen. Die befragten Proband*innen bekamen zur Unterstützung noch einmal ihre Top 4 Fotos in ausgedruckter Form vorgelegt. 


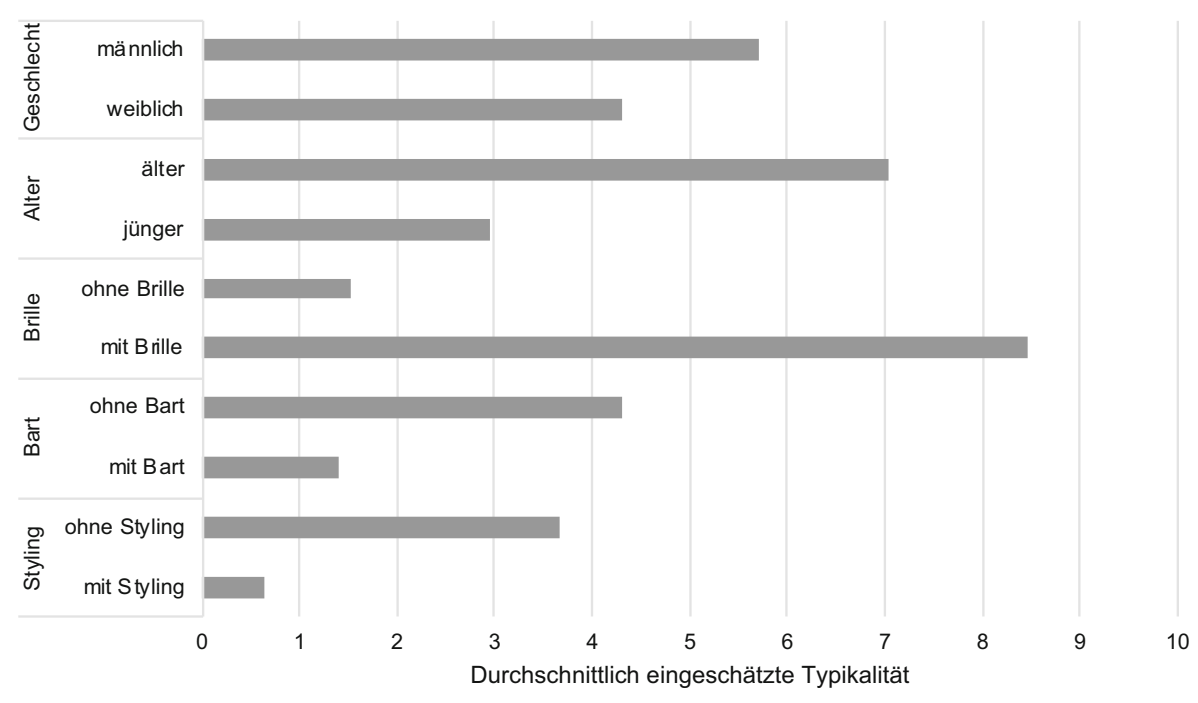

Abb. 2 Durchschnittlich eingeschätzte Typikalität der einzelnen Merkmale $(n=74)$

\section{Ergebnisse}

\subsection{Typische Merkmale von Wissenschaftler*innen}

Um zu untersuchen, welche Bedeutung die ausgewählten Merkmale für die von Schüler*innen eingeschätzte Typikalität von Wissenschaftler*innen haben (Forschungsfrage 1), wurden drei ANOVAs mit Messwiederholung durchgeführt. Um die Bedeutung des Faktors Geschlecht und mögliche Interaktionen mit den Faktoren Alter und Brille zu untersuchen, wurde eine ANOVA mit den drei Faktoren Geschlecht, Alter und Brille als Within-Faktoren bzw. unabhängigen Variablen durchgeführt. Zusätzlich wurden zwei weitere ANOVAs durchgeführt, um die Bedeutung der Faktoren Bart bei Männern und Styling bei Frauen zu untersuchen. Dabei bildeten die drei Faktoren Alter, Brille und Bart bzw. Styling die WithinFaktoren. Die von den Schüler*innen eingeschätzte Typikalität von Wissenschaftler*innen wurde bei allen drei Varianzanalysen als abhängige Variable festgelegt.

Deskriptive Statistiken (siehe Onlinematerial 1) zeigen, dass die Schüler*innen die Typikalität am höchsten für den älteren Mann mit Brille und ohne Bart einschätzten $(M=2,69, S D=1,68)$, gefolgt von der älteren Frau, ebenfalls mit Brille und ohne Styling $(M=2,03, S D=1,49)$. Am typischsten für Wissenschaftler*innen wurde das Merkmal ,mit Brille“ eingeschätzt $(M=8,47, S D=2,08)$ (siehe Abb. 2). Die zweit höchsten Typikalitätswerte erzielte das Merkmal ,älter“ $(M=7,04, S D=1,04)$, gefolgt von den Merkmalen „,männlich“ $(M=5,70, S D=1,52)$, ,ohne Bart“ $(M=4,31$, $S D=2,39)$ und „ohne Styling“ ( $M=3,66, S D=2,10)$.

Die Ergebnisse der Varianzanalysen zeigen signifikante Haupteffekte aller fünf Faktoren (siehe Tab. 2). Bezüglich des Faktors Brille zeigt sich der größte Effekt. Personen mit Brille wurden dabei typischer als Wissenschaftler*innen eingeschätzt als Personen ohne Brille. Außerdem wurden ältere Personen eher als Wissenschaft- 
Tab. 2 Haupteffekte

\begin{tabular}{llll}
\hline & $F(1,73)$ & $p$ & $\eta_{\mathrm{p}}{ }^{2}$ \\
\hline Geschlecht $^{\mathrm{a}}$ & 15,93 & $<0,001$ & 0,18 \\
Alter $^{\mathrm{a}}$ & 157,43 & $<0,001$ & 0,68 \\
Brille $^{\mathrm{a}}$ & 207,21 & $<0,001$ & 0,74 \\
Bart $^{\mathrm{b}}$ & 31,97 & $<0,001$ & 0,31 \\
Styling $^{\mathrm{c}}$ & 67,63 & $<0,001$ & 0,48 \\
\hline
\end{tabular}

${ }^{\mathrm{a}}$ Ergebnisse der ANOVA zu beiden Geschlechtern

${ }^{b}$ Ergebnisse der ANOVA zu Männern

${ }^{c}$ Ergebnisse der ANOVA zu Frauen

Tab. 3 Zweifachinteraktionen

\begin{tabular}{|c|c|c|c|}
\hline & $F(1,73)$ & $p$ & $\eta_{\mathrm{p}}^{2}$ \\
\hline Geschlecht $*$ Alter $^{\mathrm{a}}$ & 33,37 & $<0,001$ & 0,31 \\
\hline Geschlecht $*$ Brille $^{a}$ & 6,86 & 0,011 & 0,09 \\
\hline Alter $*$ Brille $^{\mathrm{a}}$ & 57,18 & $<0,001$ & 0,44 \\
\hline Alter $*$ Bart $^{\mathrm{b}}$ & 18,21 & $<0,001$ & 0,20 \\
\hline Alter $*$ Styling ${ }^{\mathrm{c}}$ & 11,66 & 0,01 & 0,14 \\
\hline Brille * Bart $^{\mathrm{b}}$ & 14,62 & $<0,001$ & 0,17 \\
\hline Brille $*$ Styling ${ }^{\mathrm{c}}$ & 35,25 & $<0,001$ & 0,33 \\
\hline
\end{tabular}

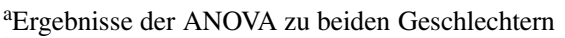

${ }^{\text {b}}$ Ergebnisse der ANOVA zu Männern

${ }^{c}$ Ergebnisse der ANOVA zu Frauen

ler*innen eingeschätzt als jüngere Personen. Frauen ohne Styling bzw. Männer ohne Bart wurden zudem eher als Wissenschaftler*innen angesehen als Frauen mit Styling bzw. Männer mit Bart. Bezüglich des Faktors Geschlecht lässt sich der geringste Effekt beobachten. Männliche Personen wurden gegenüber weiblichen Personen typischer als Wissenschaftler*innen von den Schüler*innen eingeschätzt.

Neben den signifikanten Haupteffekten zeigen die Ergebnisse sieben signifikante Zweifachinteraktionen (siehe Tab. 3): Vor allem der ältere Mann wurde von den Schüler*innen typisch als Wissenschaftler eingeschätzt. Im Gegensatz dazu wurden bei jüngeren Personen beide Geschlechter etwa gleich typisch eingeschätzt. Zudem erzielten insbesondere männliche Personen mit Brille hohe Typikalitätswerte, wohingegen sowohl männliche wie auch weibliche Personen ohne Brille eher weniger als typische Wissenschaftler*innen angesehen wurden. Die Ergebnisse zeigen außerdem, dass insbesondere ältere Personen mit Brille von den Schüler*innen als typische Wissenschaftler*innen betrachtet wurden und sowohl ältere wie auch jüngere Personen ohne Brille eher geringe Typikalitätswerte erzielten. Der ältere Mann ohne Bart sowie die ältere Frau ohne Styling wurden von den Schüler*innen verstärkt als typische Wissenschaftler*innen und sowohl der ältere wie auch jüngere Mann mit Bart bzw. die ältere wie auch jüngere Frau mit Styling eher weniger typisch eingeschätzt. Männer mit Brille und ohne Bart bzw. Frauen mit Brille und ohne Styling wurden von den Schüler*innen ebenfalls als typische Wissenschaftler*innen angesehen, wohingegen vor allem Männer ohne Brille und mit Bart bzw. Frauen ohne Brille und mit Styling die geringsten Typikalitätswerte erzielten. 
Tab. 4 Dreifachinteraktionen

\begin{tabular}{llll}
\hline & $F(1,73)$ & $p$ & $\eta_{\mathrm{p}}{ }^{2}$ \\
\hline Geschlecht $^{*}$ Alter $*$ Brille $^{\mathrm{a}}$ & 7,18 & 0,009 & 0,09 \\
Alter $*$ Brille * Bart $^{\mathrm{b}}$ & 6,33 & 0,014 & 0,08 \\
Alter $*$ Brille * Styling $^{\mathrm{c}}$ & 4,35 & 0,041 & 0,06 \\
\hline
\end{tabular}

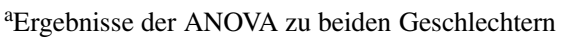

${ }^{\mathrm{b}}$ Ergebnisse der ANOVA zu Männern

${ }^{c}$ Ergebnisse der ANOVA zu Frauen

Die Ergebnisse zeigen zudem drei signifikante Dreifachinteraktionen (siehe Tab. 4). Vor allem der ältere Mann mit Brille, gefolgt von der älteren Frau ebenfalls mit Brille, wurden als typische*r Wissenschaftler*in von den Schüler*innen eingeschätzt. Weniger typisch wurden hingegen sowohl die jüngere Frau ohne Brille wie auch der jüngere Mann ohne Brille eingeschätzt. Zudem wurden insbesondere ältere Personen mit Brille und ohne Bart bzw. Styling als typische Wissenschaftler*innen eingeschätzt. Im Gegensatz dazu erzielten sowohl ältere wie auch jüngere Personen ohne Brille und mit Bart bzw. Styling nur sehr geringe Typikalitätswerte.

Zusammenfassend zeigt sich, dass vor allem die Merkmale „mit Brille“, ,älter“ und „ohne Styling“ (bei Frauen) bzw. „ohne Bart“ (bei Männern) aus Schülersicht typisch für Wissenschaftler*innen sind. Mit Blick auf den Faktor Geschlecht wurden männliche Personen verstärkt als typische Wissenschaftler eingeschätzt. Am typischsten wurde der ältere Mann mit Brille und ohne Bart, gefolgt von der älteren Frau, ebenfalls mit Brille und ohne Styling, eingeschätzt.

\subsection{Gründe für die Auswahl bestimmter, aus Schülersicht typischer Merkmale von Wissenschaftler*innen}

Zur Beantwortung der Forschungsfrage 2, wie Schüler*innen die Auswahl bestimmter, aus ihrer Sicht für Wissenschaftler*innen typischer Merkmale begründen, wurden insgesamt 18 Schüler*innen (61,1\% männlich, 27,8\% weiblich, 5,6\% divers, $M=14,29$ Jahre, $S D=0,99)$ befragt. Um ein möglichst differenziertes Bild über die Gründe von Schüler*innen für die Auswahl bestimmter Merkmale zu gewinnen, wurden sowohl Schüler*innen ausgewählt, die die stereotype Vorstellung eines Wissenschaftlers als älteren Mann mit Brille und Bart (z. B. Chambers 1983) vertraten bzw. verstärkt stereotype Merkmale ausgewählt hatten $(n=15)$, als auch Schüler*innen, die auch weniger stereotype Merkmale (z. B. Personen ohne Brille oder jüngere Personen) ausgewählt hatten $(n=3)$.

Von den befragten Schüler*innen, die die stereotype Vorstellung vertraten bzw. verstärkt stereotype Merkmale auswählten, wurde am häufigsten das Merkmal ,,mit Brille“ genannt $(n=13)$. Als Begründungen für ein Foto einer Person mit Brille führten die Schüler*innen unter anderem folgende Argumente an: „Brillenträger sehen schlauer aus“, „Brille passiert, weil man Bücher liest“" oder „Wenn man Wissenschaftler-Fotos sieht, haben die auch immer eine Brille auf.“ 12 der befragten Schüler*innen bezogen sich explizit auf das Merkmal ,älter“ und begründeten ihre Entscheidung unter anderem damit, dass Wissenschaftler*innen einen größeren Erfahrungsschatz aufweisen oder eine längere Ausbildungszeit durchlaufen: „Ältere 
haben mehr Erfahrung“ oder „Menschen mit höherem Wissensstand sind älter, weil sie eine längere Fortbildungszeit hatten“. Sechs der Schüler*innen benannten explizit das Merkmal „ohne Styling“ bei Frauen und führten als Begründungen an, dass Wissenschaftlerinnen keine Zeit hätten, sich zu stylen, und dass Schmuck bei ihrer Arbeit eher hinderlich sei: „Wenn man sich schminkt, muss man viel Zeit investiert haben, auch viel Zeit für Mode. Das passt nicht zu Wissenschaftlern.“, „Schmuck kann man beim Experimentieren nicht tragen“ oder ,Wissenschaftler haben nicht so viel Zeit sich zu schminken, sind viel beschäftigt“. Die befragten Schüler*innen, die die stereotype Vorstellung eines Wissenschaftlers als älteren Mann mit Brille und Bart vertraten, bezogen sich explizit auf das Merkmal „mit Bart“ und begründeten ihre Auswahl unter anderem wie folgt: „Kenne ich selber auch aus Filmen, dass die einen langen Bart haben.“, „Bart ab machen ist Zeitverschwendung“ oder „Von vielen Wissenschaftlern kennt man das mit Bart (z. B. Albert Einstein, Galileo Galilei).“ Lediglich eine Person bezog sich explizit auf das Merkmal „männlich“ und führte als Begründung ,aus Instinkt“ an.

Die befragten Schüler*innen, die weniger stereotype Vorstellungen vertraten, indem sie auch nicht-stereotype Merkmale ausgewählt haben und beispielsweise ein Foto einer Person ohne Brille oder einer jüngeren Person auf den ersten Platz sortierten, hatten entweder Schwierigkeiten, ihre Entscheidung explizit zu begründen, führten Argumente wie „Bauchgefühl“ an oder begründeten ihre Entscheidung damit, sich bewusst gegen die vorherrschenden Klischees entschieden zu haben: ,,aus Prinzip gegen Vorurteile“.

Zusammenfassend zeigen die Ergebnisse der Befragung der Schüler*innen zu den Gründen ihrer Top 4 Fotoauswahl, dass die meisten befragten Schüler*innen sich auch hier auf die Merkmale „mit Brille“ (72,2\%) und ,älter“ $(66,7 \%)$ bezogen.

\subsection{Geschlechterspezifische Unterschiede in den Schülervorstellungen über Wissenschaftler*innen}

Um zu untersuchen, ob sich geschlechterspezifische Unterschiede in den Schülervorstellungen über Wissenschaftler*innen feststellen lassen (Forschungsfrage 3), wurden drei Mixed ANOVAs mit jeweils drei der fünf ausgewählten Faktoren als WithinFaktoren (Geschlecht, Alter, Brille; Alter, Brille, Bart; Alter, Brille, Styling), dem Geschlecht der Schüler*innen als Between-Faktor und der eingeschätzten Typikalität als abhängige Variable durchgeführt.

Die deskriptiven Statistiken (siehe Abb. 3; Onlinematerial 2 und 3) zeigen, dass sich die von den männlichen Schülern und weiblichen Schülerinnen eingeschätzten Typikalitäten der beiden Faktoren Geschlecht (der Wissenschaftler*innen) und Bart bzw. Styling am stärksten unterscheiden: Männliche Schüler schätzten verstärkt männliche Personen als typische Wissenschaftler ein, wohingegen weibliche Schülerinnen beide Geschlechter etwa gleich typisch als Wissenschaftler*innen ansahen. Weibliche Personen erzielten bei den weiblichen Schülerinnen durchschnittlich sogar leicht höhere Typikalitätswerte als männliche Personen. Auch das Merkmal „ohne Styling“ wurde verstärkt von weiblichen Schülerinnen als typisch für Wissenschaftler*innen eingeschätzt, wohingegen das Merkmal ,mit Bart“ bei männlichen Schülern höhere Typikalitätswerte erzielte. Die deskriptiven Statistiken zeigen 


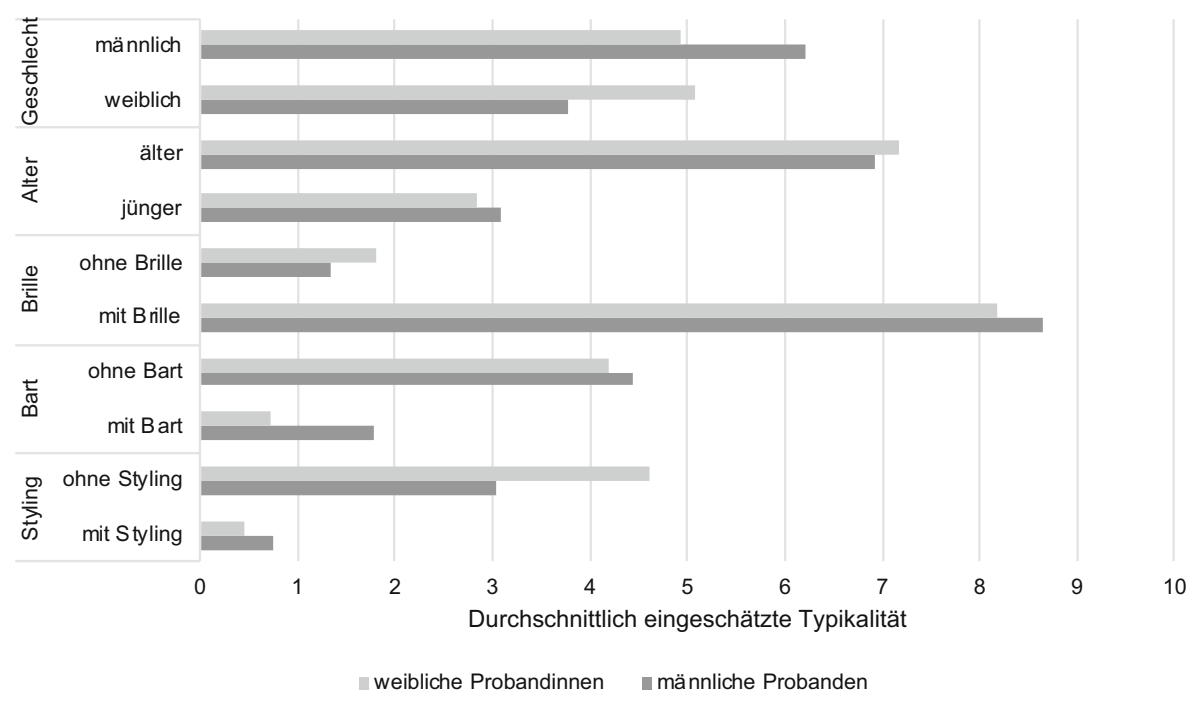

Abb. 3 Durchschnittlich eingeschätzte Typikalität der einzelnen Merkmale getrennt nach Probandengeschlecht (weiblich: $n=26$; männlich: $n=46$ )

außerdem, dass Personen mit Brille höhere Typikalitätswerte bei den männlichen Schülern erzielten, wohingegen ältere Personen verstärkt von weiblichen Probandinnen als typische Wissenschaftler*innen eingeschätzt wurden.

Die Ergebnisse der Varianzanalysen (siehe Tab. 5) bestätigen diese deskriptiven Unterschiede zum Teil und zeigen signifikante Interaktionen zwischen dem Geschlecht der Schüler*innen und dem Faktor Geschlecht (der Wissenschaftler*innen) sowie zwischen dem Geschlecht der Schüler*innen und dem Faktor Styling. Zudem zeigen die Ergebnisse eine signifikante Dreifachinteraktion zwischen dem Geschlecht der Schüler*innen und den Faktoren Brille und Styling: Vor allem weibliche Personen mit Brille und ohne Styling wurden verstärkt von weiblichen Schülerinnen als typische Wissenschaftlerinnen angesehen. Weitere signifikante Interaktionen konnten nicht festgestellt werden.

\subsection{Zusammenhänge zwischen den Schülervorstellungen und weiteren interessierenden Variablen}

Um zu explorieren, ob und inwiefern die Vorstellungen der Schüler*innen über Wissenschaftler*innen mit ihren mathematik- und naturwissenschaftsbezogenen Aktivitäten bzw. ihrer Freude und ihrem Interesse an Mathematik und den Naturwissenschaften zusammenhängen (Forschungsfrage 4 und 5), wurden Korrelationen berechnet. Als Maß der Effektstärke wurde Pearson's $r$ verwendet. 
Tab. 5 Ergebnisse der Mixed ANOVAs

\begin{tabular}{|c|c|c|c|}
\hline & $F(1,70)$ & $p$ & $\eta_{\mathrm{p}}^{2}$ \\
\hline Geschlecht $*$ Probandengeschlecht $^{\mathrm{a}}$ & 15,50 & $<0,001$ & 0,18 \\
\hline Alter $*$ Probandengeschlecht ${ }^{\mathrm{a}}$ & 0,51 & 0,479 & 0,01 \\
\hline Brille $*$ Probandengeschlecht ${ }^{\mathrm{a}}$ & 0,81 & 0,370 & 0,01 \\
\hline Bart $*$ Probandengeschlecht ${ }^{\mathrm{b}}$ & 0,53 & 0,467 & 0,01 \\
\hline Styling * Probandengeschlecht ${ }^{\mathrm{c}}$ & 6,13 & 0,016 & 0,08 \\
\hline Geschlecht $*$ Alter $*$ Probandengeschlecht ${ }^{\mathrm{a}}$ & 0,40 & 0,531 & 0,01 \\
\hline Geschlecht $*$ Brille $*$ Probandengeschlecht ${ }^{\mathrm{a}}$ & 2,28 & 0,135 & 0,03 \\
\hline Alter $*$ Brille $*$ Probandengeschlecht ${ }^{\mathrm{a}}$ & 0,07 & 0,798 & 0,00 \\
\hline Alter $*$ Bart $*$ Probandengeschlecht ${ }^{\mathrm{b}}$ & 0,53 & 0,469 & 0,01 \\
\hline Alter $*$ Styling $*$ Probandengeschlecht ${ }^{\mathrm{c}}$ & 0,50 & 0,484 & 0,01 \\
\hline Brille $*$ Bart $*$ Probandengeschlecht ${ }^{\mathrm{b}}$ & 0,19 & 0,664 & 0,00 \\
\hline Brille $*$ Styling $*$ Probandengeschlecht ${ }^{\mathrm{c}}$ & 7,21 & 0,009 & 0,09 \\
\hline Geschlecht $*$ Alter $*$ Brille $*$ Probandengeschlecht ${ }^{\mathrm{a}}$ & 0,55 & 0,460 & 0,01 \\
\hline Alter $*$ Brille $*$ Bart $*$ Probandengeschlecht ${ }^{\mathrm{b}}$ & 0,11 & 0,744 & 0,00 \\
\hline Alter $*$ Brille $*$ Styling $*$ Probandengeschlecht ${ }^{\mathrm{c}}$ & 0,76 & 0,386 & 0,01 \\
\hline
\end{tabular}

${ }^{\text {a}}$ Ergebnisse der Mixed ANOVA zu beiden Geschlechtern

${ }^{\mathrm{b}}$ Ergebnisse der Mixed ANOVA zu Männern

${ }^{\mathrm{c}}$ Ergebnisse der Mixed ANOVA zu Frauen

\subsubsection{Zusammenhänge zwischen den Schülervorstellungen mit den mathematik- und naturwissenschaftsbezogenen Aktivitäten}

Mit Blick auf die mathematikbezogenen Aktivitäten der Schüler*innen zeigen die Ergebnisse einen schwach positiven Zusammenhang zwischen den mathematikbezogenen Aktivitäten und der eingeschätzten Typikalität des Merkmals ,mit Bart“ $(r=0,25, p=0,030)$. Das Merkmal ,mit Bart“ wurde somit verstärkt von Schüler*innen mit eher hohen mathematikbezogenen Aktivitäten typisch für Wissenschaftler eingeschätzt. Außerdem belegen die Ergebnisse schwache und nicht signifikante Zusammenhänge zwischen den mathematikbezogenen Aktivitäten der Schüler*innen und den eingeschätzten Typikalitäten der Merkmale ,älter“ $(r=-0,15$, $p=0,191)$, „männlich“ $(r=0,15, p=0,215)$, ,ohne Bart“ $(r=-0,15, p=0,200)$ und „ohne Styling“ ( $r=-0,13, p=0,277)$. Bezüglich des Faktors Brille sowie des Merkmals ,mit Styling“ offenbaren die Ergebnisse keinen Zusammenhang zwischen den mathematikbezogenen Aktivitäten und den von den Schüler*innen eingeschätzten Typikalitäten dieses Faktors bzw. Merkmals (Brille: $r=0,00, p=0,976$; ,mit Styling“: $r=0,04, p=0,757)$.

Auch im Hinblick auf die naturwissenschaftsbezogenen Aktivitäten zeigen die Ergebnisse schwache und nicht signifikante Zusammenhänge zwischen den naturwissenschaftsbezogenen Aktivitäten der Schüler*innen und den eingeschätzten Typikalitäten der Merkmale ,männlich“ ( $r=0,20, p=0,089)$ und ,ohne Styling“ ( $r=-0,11$, $p=0,365)$. Zusammenhänge zwischen den naturwissenschaftsbezogenen Aktivitäten und den eingeschätzten Typikalitäten der anderen Faktoren bzw. Merkmale wurden nicht festgestellt (Alter: $r=0,06, p=0,607$; Brille: $r=0,05, p=0,701$; ,,mit 
Bart": $r=0,08, p=0,474$; ,ohne Bart": $r=0,05, p=0,705$; „mit Styling“: $r=-0,06$, $p=0,624)$.

\subsubsection{Zusammenhänge zwischen den Schülervorstellungen mit der Freude und dem Interesse der Schüler*innen an Mathematik und den Naturwissenschaften}

Hinsichtlich der Freude und des Interesses der Schüler*innen an Mathematik belegen die Ergebnisse der berechneten Korrelationen einen schwach negativen Zusammenhang zwischen der Freude und dem Interesse der Schüler*innen an Mathematik und der eingeschätzten Typikalität des Merkmals ,älter“ ( $r=-0,29, p=0,014)$. Das Merkmal ,älter“ wurde somit verstärkt von Schüler*innen mit eher geringer Freude und geringem Interesse an Mathematik typisch für Wissenschaftler*innen eingeschätzt. Außerdem zeigen die Ergebnisse schwache und nicht signifikante Zusammenhänge zwischen der Freude und dem Interesse der Schüler*innen an Mathematik und den eingeschätzten Typikalitäten der Merkmale „,männlich“ $(r=0,23, p=0,050)$, ,mit Brille“ $(r=-0,13, p=0,268)$, „mit Bart“ $(r=0,19, p=0,113)$ und ,ohne Styling“ $(r=-0,22, p=0,061)$. Zwischen der Freude und dem Interesse der Schüler*innen an Mathematik und den eingeschätzten Typikalitäten der Merkmale „ohne Bart“ sowie „mit Styling“ wurden keine Zusammenhänge festgestellt (,ohne Bart“: $r=-0,03$, $p=0,775$; ,mit Styling“: $r=0,09, p=0,466$ ).

Bezüglich der Freude und des Interesses an den Naturwissenschaften zeigen die Ergebnisse ebenfalls schwache und nicht signifikanten Zusammenhänge zwischen der Freude und dem Interesse der Schüler*innen an den Naturwissenschaften und den eingeschätzten Typikalitäten der Merkmal ,männlich“ $(r=0,20, p=0,081)$, ,ohne Bart“ ( $r=0,15, p=0,195)$ sowie ,mit Styling“ $(r=-0,12, p=0,295)$. Zusammenhänge zwischen der Freude und dem Interesse an den Naturwissenschaften und den eingeschätzten Typikalitäten der anderen Faktoren bzw. Merkmale wurden nicht festgestellt (Alter: $r=0,04, p=0,708$; Brille: $r=0,02, p=0,882$; „mit Bart“: $r=-0,02$, $p=0,840 ;$, ,ohne Styling“‘: $r=-0,07, p=0,556)$.

\section{Diskussion}

Stereotype Vorstellungen über Wissenschaftler*innen - wie sie in bisherigen Studien vielfach festgestellt wurden (u. a. Mead und Métraux 1957; Chambers 1983; Hassard 1990; Boylan et al. 1992) - können vor allem für die Entwicklung positiver Einstellungen von Schüler*innen gegenüber Wissenschaft sowie für zukünftige Bildungs- und Berufsentscheidungen der Schüler*innen Konsequenzen haben (z. B. Boylan et al. 1992; Finson 2002; Buldu 2006; Laubach et al. 2012; Stamer et al. 2019). Vor diesem Hintergrund wurden in der vorliegenden Studie Vorstellungen von Schüler*innen über Wissenschaftler*innen untersucht sowie Zusammenhänge zwischen diesen Vorstellungen und ihren mathematik- und naturwissenschaftsbezogenen Aktivitäten bzw. ihrer Freude und ihrem Interesse an Mathematik und den Naturwissenschaften exploriert. Bisher wurden Schülervorstellungen über Wissenschaftler*innen häufig mit dem DAST oder modifizierten DAST-Varianten erfasst, die jedoch kritisch zu bewerten sind. Es mangelt an Messverfahren, die Aufschluss 
über die Bedeutung einzelner Merkmale für die von Schüler*innen eingeschätzte Typikalität von Wissenschaftler*innen geben können. In der vorliegenden Studie konnten durch den Einsatz einer eigens entwickelten Fotosortierungsaufgabe Vorstellungen von Schüler*innen über Wissenschaftler*innen systematisch untersucht und die Bedeutung einzelner, auf Grundlage bisheriger Befunde ausgewählter äuBerlicher Merkmale von Wissenschaftler*innen für die von den Schüler*innen eingeschätzte Typikalität herausgestellt werden.

Die Ergebnisse der Varianzanalyse im Hinblick auf Forschungsfrage 1 zeigen, dass die Faktoren Brille und Alter die größten Effekte auf die von Schüler*innen eingeschätzte Typikalität von Wissenschaftler*innen haben. Die verstärkte Auswahl der Merkmale „mit Brille“ und ,älter“ steht im Einklang mit Befunden bisheriger Studien, in denen die Schülervorstellungen über Wissenschaftler*innen schriftlich oder zeichnerisch erfasst wurden (u. a. Mead und Métraux, 1957; Chambers 1983). Die befragten Schüler*innen begründeten ihre Auswahl unter anderem damit, dass Wissenschaftler*innen eine Brille tragen und älter sind, da sie viel lesen müssten und eine längere Ausbildungszeit hätten (Forschungsfrage 2). Dieses Ergebnis spiegelt sich im folgenden Zitat, welches sich auch im Titel dieser Arbeit wiederfinden lässt und im Rahmen der Befragung der Schüler*innen nach den Gründen ihrer Top 4 Fotoauswahl entstand, wider: „Meistens sind Forscher älter, meist tragen die eine Brille“. Ein ähnliches Ergebnis lässt sich auch in der Studie von Boylan et al. (1992) beobachten, in der jüngere Personen von den befragten Schüler*innen oftmals als Lernende oder Wissenschaftler*innen in Ausbildung beschrieben wurden. Im Gegensatz zur Studie von Boylan et al. (1992) stellten die Proband*innen der vorliegenden Studie jedoch keine Verbindungen zu ihren eigenen Naturwissenschaftslehrer*innen her, sondern bezogen sich in ihren Begründungen eher auf bekannte Wissenschaftler*innen wie beispielsweise Albert Einstein oder Galileo Galilei. Außerdem wurde in den erwähnten Studien oftmals das Geschlecht „,männlich“ als Attribut der stereotypen Vorstellung herausgestellt und auch in der vorliegenden Studie wurden verstärkt männliche Personen als typische Wissenschaftler angesehen. Zudem verdeutlichen die Ergebnisse zu Forschungsfrage 3 geschlechterspezifische Unterschiede, wie sie unter anderem auch in den Studien von Fort und Varney (1989), Christidou et al. (2012) und Miller et al. (2018) festgestellt werden konnten.

Anders als in bisherigen Studien und im Gegensatz zu den Ergebnissen der mündlichen Befragung einzelner Schüler*innen, die sich explizit auf das Merkmal ,,mit Bart" bezogen haben und dieses als typisch für Wissenschaftler einschätzten, wurden jedoch in der vorliegenden Studie verstärkt männliche Personen ohne Bart als typische Wissenschaftler eingeschätzt. Dieser Widerspruch könnte möglicherweise darauf zurückgeführt werden, dass ein Teil der Schüler*innen den Bart, der beiden männlichen Fotomodellen nachträglich mithilfe eines Bildbearbeitungsprogrammes angebracht wurde, nicht authentisch für Wissenschaftler wahrgenommen haben könnten. Dieses Ergebnis deutet darauf hin, dass nicht nur das Vorhandensein eines Bartes, sondern vor allem auch das Aussehen bzw. Styling des Bartes eine wichtige Rolle für die Einschätzung der Typikalität dieses Merkmals spielt. Um den möglichen Einfluss eines Bartes für die von Schüler*innen eingeschätzte Typikalität von Wissenschaftler*innen genauer zu untersuchen, bedarf es daher weiterer Studien, in denen nicht nur zwischen dem Vorhandensein bzw. Nicht-Vorhandensein 
eines Bartes, sondern vor allem auch zwischen verschieden gestylten Bärten (z.B. gepflegt vs. eher wild) unterschieden wird.

Im Hinblick auf mögliche Zusammenhänge zwischen den Vorstellungen und den mathematik- und naturwissenschaftsbezogenen Aktivitäten der Schüler*innen (Forschungsfrage 4) zeigen die Ergebnisse der berechneten Korrelationen einen schwach positiven Zusammenhang zwischen den mathematikbezogenen Aktivitäten und der eingeschätzten Typikalität des Merkmals ,mit Bart“. Männer mit Bart wurden somit verstärkt von Schüler*innen mit eher hohen mathematikbezogenen Aktivitäten als typische Wissenschaftler eingeschätzt. Ähnlich wie die Ergebnisse der Studie von Buldu (2006) deuten auch die Ergebnisse der vorliegenden Studie darauf hin, dass die Vorstellungen von Schüler*innen über Wissenschaftler*innen von den Medien beeinflusst werden. Beispielsweise begründete ein Schüler seine Auswahl eines Mannes mit Bart als typischen Wissenschaftler damit, dass er es aus Filmen kenne, dass Wissenschaftler einen langen Bart hätten. Hinsichtlich möglicher Zusammenhänge zwischen den Vorstellungen mit der Freude und dem Interesse der Schüler*innen an Mathematik und den Naturwissenschaften (Forschungsfrage 5) zeigen die Ergebnisse der berechneten Korrelationen einen schwach negativen Zusammenhang zwischen der Freude und dem Interesse der Schüler*innen an Mathematik und der eingeschätzten Typikalität des Merkmals ,,älter“. Das Merkmal ,jünger“ wurde somit verstärkt von Schüler*innen mit eher hoher Freude und hohem Interesse an Mathematik typisch für Wissenschaftler*innen eingeschätzt. Dieses Ergebnis könnte auf den „Selbst-Prototypen-Abgleich“ (Setterlund und Niedenthal 1993; Hannover und Kessels 2002) zurückgeführt werden: Schüler*innen mit eher hoher Freude und hohem Interesse an Mathematik setzen sich eher mit möglichen wissenschaftlichen Berufsfeldern auseinander und können sich daher auch eher mit der Rolle eines*einer Wissenschaftler*in identifizieren und sich selbst diese Rolle zutrauen (vgl. Hannover und Kessels 2002; Laubach et al. 2012; Schiepe-Tiska et al. 2016a; Stamer et al. 2019). Diese wahrgenommene Ähnlichkeit der Schüler*innen mit den jeweiligen Prototypen wirkt sich wiederum auf das Interesse aus (vgl. Wentorf et al. 2015).

Zusammenfassend verdeutlichen die Ergebnisse der vorliegenden Studie die Notwendigkeit, Schülervorstellungen zu verändern und insbesondere auch gezielt Bilder von jüngeren Wissenschaftler*innen zu vermitteln, um die Identifikation von Schüler*innen mit Wissenschaftler*innen und folglich das Interesse an Wissenschaft sowie wissenschaftlichen Bildungs- und Berufswegen zu fördern (vgl. Laubach et al. 2012; Stamer et al. 2019). Auch wenn die Vorstellungen der Schüler*innen über Wissenschaftler*innen stark von den Medien geprägt werden, können im Rahmen schulischer Kontexte Möglichkeiten ergriffen werden, solch stereotype Vorstellungen zu verändern: Eine Möglichkeit, den stereotypen Vorstellungen von Schüler*innen über Wissenschaftler*innen entgegenzuwirken, könnten regelmäßige Begegnungen mit Wissenschaftler*innen darstellen (vgl. Finson 2002; Buldu 2006). Im Unterricht oder beispielsweise in Schülerlaborprojekten könnten die Schülervorstellungen über Wissenschaftler*innen überprüft werden, indem die Schüler*innen echte Wissenschaftler*innen bei ihrer Arbeit treffen. Schülerlabore gelten dabei als authentische Lernumgebungen, da sie durch die inhaltliche und methodische Gestaltung ihrer Pro- 
jekte eine Nähe zur „echten“ Arbeit von Wissenschaftler*innen herstellen (Haupt und Hempelmann 2015).

\subsection{Limitationen}

Durch den Einsatz einer eigens entwickelten Fotosortierungsaufgabe konnten in der vorliegenden Studie die Vorstellungen von Schüler*innen über Wissenschaftler*innen erstmals systematisch untersucht und die Bedeutung einzelner ausgewählter Merkmale von Wissenschaftler*innen für die von den Schüler*innen eingeschätzte Typikalität herausgestellt werden. In Bezug auf die verwendete Methode ergeben sich einige Limitationen. Zwar werden „Wissenschaft“ und „Wissenschaftler*innen“ meist mit MINT-Disziplinen und naturwissenschaftlichen Arbeitsweisen assoziiert (vgl. Nachtigall et al. 2018; Ziegler et al. 2018; Stamer et al. 2019) und auch die Schüleraussagen - beispielsweise die Nennung von Albert Einstein und Galileo Galilei oder die Aussage, dass Schmuck beim Experimentieren hinderlich sei - deuten darauf hin, dass die befragten Schüler*innen sich auf Naturwissenschaftler*innen bezogen haben, allerdings kann nicht davon ausgegangen werden, dass alle Schüler*innen im Rahmen der Fotosortierungsaufgabe an Naturwissenschaftler*innen gedacht haben. Vor allem vor dem Hintergrund des Einflusses der Vorstellungen auf die Bildungs- und Berufswahl der Jugendlichen wäre es notwendig die vorliegende Studie mit einem Instrument zu replizieren, welches die Vorstellungen von Schüler*innen expliziter und in Bezug auf Wissenschaftler*innen bestimmter Disziplinen, wie Mathematik und/oder Naturwissenschaften, erfasst. Zudem kann mithilfe der Fotosortierungsaufgabe nicht die gesamte Bandbreite der Vorstellungen von Schüler*innen über Wissenschaftler*innen erfasst werden. Durch die Festlegung auf bestimmte stereotype Merkmale von Wissenschaftler*innen wird den Schüler*innen - im Gegensatz zum bisher vielfach verwendeten DAST oder modifizierten DASTVarianten - keine Möglichkeit gegeben, eigene Merkmale zu ergänzen. Zudem scheinen einige Proband*innen Teile der Konstruktionslogik durchschaut zu haben, indem sie sich bewusst gegen die vorherrschenden Stereotype entschieden haben, was ihre Auswahl der Top 4 Fotos und folglich die Ergebnisse der vorliegenden Studie beeinflusst haben könnte. Die systematische Variation der Faktoren und Merkmale ermöglicht allerdings, Aussagen über die Bedeutung einzelner Merkmale für die von Schüler*innen eingeschätzte Typikalität von Wissenschaftler*innen treffen zu können. Folglich sollte die hier vorgestellte Methode nicht in Konkurrenz, sondern in Ergänzung zu bisherigen Methoden gesehen werden. Eine weitere potenzielle Limitation besteht bezüglich der Interviews, in denen einzelne Schüler*innen abschließend zu den Gründen ihrer Top 4 Fotoauswahl befragt wurden, da die befragten Schüler*innen eventuell nicht mehr alle Gedanken erinnern oder rekonstruieren und nur das verbalisieren konnten, dessen sie sich bewusst waren. In weiteren Studien erscheinen daher auch offene Interviews sinnvoll. Eine weitere Problematik besteht bezüglich der Zusammensetzung der Stichprobe der hier berichteten Arbeit. Die ungleiche Verteilung der Geschlechter der Proband*innen könnte die Ergebnisse verzerren. Wie in zahlreichen anderen Studien zu Schülervorstellungen über Wissenschaftler*innen wurde in der vorliegenden Studie ebenfalls ein äußeres Bild (in Form eines Fotos) als Indikator für die Vorstellung einer Person verwendet, wodurch nur 
ein Teil der Vorstellung sichtbar wird. Die Vorstellung von Wissenschaftler*innen umfasst nicht nur das Äußere, sondern beispielsweise auch nicht sichtbare innere Werte und Einstellungen (vgl. bspw. Höttecke 2001).

\subsection{Fazit und Implikationen}

In der vorliegenden Arbeit konnte durch die systematische Variation ausgewählter Merkmale im Rahmen der Fotosortierungsaufgabe erstmals auch die Bedeutung einzelner stereotyper Merkmale von Wissenschaftler*innen für die Vorstellungen von Schüler*innen untersucht werden. Es zeigte sich, dass vor allem die Faktoren Brille und Alter große Effekte auf die von Schüler*innen eingeschätzte Typikalität von Wissenschaftler*innen haben. Zudem bestätigen die Ergebnisse die in bisherigen Studien festgesellten geschlechterspezifischen Unterschiede. Die vorliegende Studie liefert außerdem Hinweise darauf, dass die Vorstellungen von Schüler*innen über Wissenschaftler*innen mit Darstellungen von (bekannten) Wissenschaftler*innen in den Medien und mit der persönlichen Identifizierung mit der Rolle eines*einer Wissenschaftler*in zusammenhängen. Folglich gewinnt die Förderung authentischer Schülervorstellungen über Wissenschaftler*innen - vor allem auch vor dem Hintergrund der Generierung wissenschaftlichen Nachwuchses - an Bedeutung. Noch offen bleibt, welche Vorstellungen Schüler*innen vom Charakter und von den inneren Werten von Wissenschaftler*innen haben und welchen Einfluss diese Vorstellungen auf die Wahrnehmung der Schüler*innen von Wissenschaft haben. Außerdem sollte zukünftig weiter untersucht werden, mit welchen Variablen die Schülervorstellungen über Wissenschaftler*innen zusammenhängen, um die Bedeutung solch stereotyper Vorstellungen herausstellen und Interventionen zur Förderung angemessener Vorstellungen ableiten zu können.

Zusatzmaterial online Zusätzliche Informationen sind in der Online-Version dieses Artikels (https://doi. org/10.1007/s42010-021-00110-1) enthalten.

Danksagung Die vorliegende Studie wurde innerhalb des Promotionskollegs „Metakognitives Monitoring in authentischen Lehr-/Lernkontexten im Schülerlabor“ (MeMo-akS) durch die Professional School of Education (PSE) der Ruhr-Universität Bochum gefördert. Wir bedanken uns ganz herzlich bei allen Mitgliedern des Kollegs, den Fotomodellen und allen an dieser Studie beteiligten Schulklassen sowie dem Alfried Krupp-Schülerlabor der Ruhr-Universität Bochum. Außerdem danken wir den anonymen Gutachter*innen für die wertvollen Hinweise, die in die Überarbeitung des Manuskripts eingeflossen sind.

Funding Open Access funding enabled and organized by Projekt DEAL.

Open Access Dieser Artikel wird unter der Creative Commons Namensnennung 4.0 International Lizenz veröffentlicht, welche die Nutzung, Vervielfältigung, Bearbeitung, Verbreitung und Wiedergabe in jeglichem Medium und Format erlaubt, sofern Sie den/die ursprünglichen Autor(en) und die Quelle ordnungsgemäß nennen, einen Link zur Creative Commons Lizenz beifügen und angeben, ob Änderungen vorgenommen wurden.

Die in diesem Artikel enthaltenen Bilder und sonstiges Drittmaterial unterliegen ebenfalls der genannten Creative Commons Lizenz, sofern sich aus der Abbildungslegende nichts anderes ergibt. Sofern das betreffende Material nicht unter der genannten Creative Commons Lizenz steht und die betreffende Handlung nicht nach gesetzlichen Vorschriften erlaubt ist, ist für die oben aufgeführten Weiterverwendungen des Materials die Einwilligung des jeweiligen Rechteinhabers einzuholen. 
Weitere Details zur Lizenz entnehmen Sie bitte der Lizenzinformation auf http://creativecommons.org/ licenses/by/4.0/deed.de.

\section{Literatur}

Boylan, C. R., Hill, D. M., Wallace, A.R., \& Wheeler, A.E. (1992). Beyond Stereotypes. Science Education, 76(5), 465-476.

Buldu, M. (2006). Young children's perceptions of scientists: a preliminary study. Educational Research, 48(1), 121-132.

Chambers, D. W. (1983). Stereotypic images of the scientist: the draw-A-scientist test. Science Education, $67(2), 255-265$.

Christidou, V. (2011). Interest, attitudes and images related to science: combining students' voices with the voices of school science, teachers, and popular science. International Journal of Environment and Science Education, 6(2), 141-159.

Christidou, V., Hatzinikita, V., \& Samaras, G. (2012). The image of scientific researchers and their activity in Greek adolescents' drawings. Public Understanding of Science, 21(5), 626-647.

Cicchetti, D. V., \& Sparrow, S. S. (1990). Assesment of adaptive behavior in young children. In J. J. Johnson \& J. Goldman (Hrsg.), Developmental assessment in clinical child psychology. A handbook (S. 173-196). New York: Pergamon.

Driver, R., Leach, J., Millar, R., \& Scott, P. (1996). Young people's images of science. Buckingham: Open University Press.

Farland-Smith, D. (2012). Development and field test of the modified draw-a-scientist test and the draw-ascientist rubric. School Science and Mathematics, 112, 109-116.

Finson, K. D. (2002). Drawing a scientist: what we do and do not know after fifty years of drawings. School Science and Mathematics, 102(7), 335-345.

Finson, K.D., \& Pederson, J. (2011). What are visual data and what utility do they have in science education? Journal of Visual Literacy, 30(1), 65-84.

Finson, K.D., Beaver, J.B., \& Cramond, B.L. (1995). Development and field test of a checklist for the draw-a-scientist test. School Science and Mathematics, 95, 195-205.

Fort, D.C., \& Varney, H.L. (1989). How students see science: Mostly male, mostly white, and mostly benevolent. Science and Children, 26(8), 8-13.

Hannover, B., \& Kessels, U. (2002). Challenge the science-stereotype! Der Einfluss von Technikfreizeitkursen auf das Naturwissenschaften-Stereotyp von Schülerinnen und Schülern. Zeitschrift für Pädagogik, 45, 341-358.

Hassard, J. (1990). Science experiences. Menlo Park: Addison-Wesley.

Haupt, O. J., \& Hempelmann, R. (2015). Schülerlabore in Art und Form - Eine Typensache! In LernortLabor (Hrsg.), Schülerlabor-Atlas 2015. Schülerlabore im deutschsprachigen Raum (S. 14-21). Stuttgart: Klett MINT.

Höttecke, D. (2001). Die Vorstellungen von Schülern und Schülerinnen von der „Natur der Naturwissenschaften“. Zeitschrift für die Didaktik der Naturwissenschaften, 7, 7-23.

Labudde, P., \& Möller, K. (2012). Stichwort: Naturwissenschaftlicher Unterricht. Zeitschrift für Erziehungswissenschaft, 15, 11-36.

Laubach, T. A., Crofford, G. D., \& Marek, E. A. (2012). Exploring native American students' perceptions of scientists. International Journal of Science Education, 34(11), 1769-1794.

Losh, S. C., Wilke, R., \& Pop, M. (2008). Some methodological issues with "draw a scientist test" among young children. International Journal of Science Education, 30, 773-792.

Mang, J., Ustjanzew, N., Leßke, I., Schiepe-Tiska, A., \& Reiss, K. (2019). PISA 2015 Skalenhandbuch. Dokumentation der Erhebungsinstrumente. Münster: Waxmann.

McNay, M. (1988). Children's views of science. Crucible, 19, 13-15.

Mead, M., \& Métraux, R. (1957). Image of the scientist among high-school students. A pilot study. Science, 126(3270), 384-390.

Miller, D. I., Nolla, K.M., Eagly, A.H., \& Uttal, D.H. (2018). The development of children's genderscience stereotypes: a meta-analysis of 5 decades of U.S. draw-a-scientist studies. Child Development, 89(6), 1943-1955.

Nachtigall, V., Rummel, N., \& Serova, K. (2018). Authentisch ist nicht gleich authentisch - Wie Schülerinnen und Schüler die Authentizität von Lernaktivitäten im Schülerlabor einschätzen. Unterrichtswissenschaft, 46, 299-319. 
Osborne, J., Simon, S., \& Collins, S. (2003). Attitudes towards science: a review of the literature and its implications. International Journal of Science Education, 25(9), 1049-1079.

Schiepe-Tiska, A., Roczen, Müller, K., Prenzel, M., \& Osborne, J. (2016a). Science-related outcomes: attitudes, motivation, value beliefs, strategies. In S. Kuger, E. Klieme, N. Jude \& D. Kaplan (Hrsg.), Assessing contexts of learning. An international perspective (S. 301-329). Cham: Springer.

Schiepe-Tiska, A., Rönnebeck, S., Schöps, K., Neumann, K., Schmidtner, S., Parchmann, I., \& Prenzel, M. (2016b). Naturwissenschaftliche Kompetenz in PISA 2015 - Ergebnisse des internationalen Vergleichs mit einem modifizierten Testansatz. In K. Reiss, C. Sälzer, A. Schiepe-Tiska, E. Klieme \& O. Köller (Hrsg.), PISA 2015. Eine Studie zwischen Kontinuität und Innovation (S. 45-98). Münster: Waxmann.

Schiepe-Tiska, A., Simm, I., \& Schmidtner, S. (2016c). Motivationale Orientierungen, Selbstbilder und Berufserwartungen in den Naturwissenschaften in PISA 2015. In K. Reiss, C. Sälzer, A. SchiepeTiska, E. Klieme \& O. Köller (Hrsg.), PISA 2015. Eine Studie zwischen Kontinuität und Innovation (S. 99-132). Münster: Waxmann.

Setterlund, M. B., \& Niedenthal, P. M. (1993). „Who am I? Why am I here?“ Self-esteem, self-clarity, and prototype matching. Journal of Personality and Social Psychology, 65(4), 769-780.

Stamer, I., Kubsch, M., Steiner, M., Schwarzer, S., \& Parchmann, I. (2019). Scientists, their work, and how others perceive them: self-perceptions of scientists and students' stereotypes. RISTAL, 2, 85-101.

Thomson, M. M., Zakaria, Z., \& Radut-Taciu, R. (2019). Perceptions of scientists and stereotypes through the eyes of young school children. Education Research International. https://doi.org/10.1155/2019/ 6324704.

Wentorf, W., Höffler, T.N., \& Parchmann, I. (2015). Schülerkonzepte über das Tätigkeitsspektrum von Naturwissenschaftlerinnen und Naturwissenschaftlern: Vorstellungen, korrespondierende Interessen und Selbstwirksamkeitserwartungen. Zeitschrift für Didaktik der Naturwissenschaften, 21, 207-222.

Ziegler, R., Kremer, B., \& Weißkopf, M. (2018). Medizin und neue Technologien, Analysen und Erkenntnisse, Intelligenz und Ausdauer - Welche Vorstellung hat die Bevölkerung von Wissenschaft und Forschenden? Ergebnisse der offenen Fragestellungen im Wissenschaftsbarometer 2017. Berlin: Wissenschaft im Dialog. 